\title{
Importance, Implementation and Limitation of Pharma Doctors in Clinical Pharmacy Practice for Out-patient Health Care Setting
}

\author{
Allu Harikrishna \\ Marketing Officer, Bureau of Pharma PSUs of India, New Delhi, INDIA.
}

\begin{abstract}
Pharma Doctors importance, Implementation and limitation for practicing clinical Pharmacy services for out-patient at the site of community Pharmacy. Clinical Pharmacy services were most important service for out-patients like DM, HTN, CKD, Psychiatric patients, CHD and co-morbidity Disease patients to avoid drug relevant problems for the safest and affective therapy. Providing a Disease and Medication knowledge, Drug and Poison information to the patents, Public and Health care Professionals. Aim: To the perform "Importance, Implementation and limitation of Pharma Doctors in Clinical Pharmacy Practice for Out-Patient Health care setting". For providing safest effective prescription order. Clinical Pharmacy establishment at the site of community Pharmacy: Clinical Pharmacy Service is a part of dispensary service as interpretation, evaluation and implementation of prescription these service will give safest and effective therapeutic prescription base on these terms need to establish clinical Pharmacy in the site of Community Pharmacy. Conclusion: Clinical Pharmacy Service will help the patient to provide safest and effective therapeutic prescription and lowering the health economic burden, providing the best medication administration knowledge to the patient for avoiding medication non-adherence.
\end{abstract}

Key words: Clinical Pharmacy, Community Pharmacy, Dispensing, safety and Effective therapy, Pharmaco-econamic efficacy, Pharmacy Practice regulation, Clinical establishment act, Pharmacy act, Drug and Poison information, Patient Counseling, Drug related Problems.

\section{INTRODUCTION}

Doctor of Pharmacy (Pharm.D) Graduates generally call as a "Pharma Doctors" these are professionally called as a Clinical Pharmacists these course are introduced by the Pharmacy Council of India (PCI). ${ }^{1}$ Clinical Pharmacy Practice means the pharmacists, who use their skills and knowledge to take an active participation in patient health, through effective interaction and communication with both patients and other healthcare professionals in a health care setting. ${ }^{2}$ Clinical Pharmacy Services is necessary in community for providing better health care services by using clinical skills of Clinical
Pharmacist in out-Patients. ${ }^{3}$ (Clinical Pharmacist role in health care system was providing patient care that analyzing the use of medication and health promotion, wellness and disease prevention. Clinical Pharmacists Patient care was require in all health care system. Clinical Pharmacists need to maintain good communication with all health care professionals. ${ }^{4}$ Now a days the role of Pharmacist was changed In India as a Clinical Pharmacy practitioner need to work with Health care Professional, monitoring the Pharmacotherapetic efficacy of the Patient, Health care safety, Cost effective Therapy Monitoring, Patient education on Medication and Disease, Drug and Poison
DOI: 10.5530/ijopp.14.4.49

Address for correspondence: Dr. Allu Harikrishna, Marketing Officer, Bureau of Pharma PSUs of India, New Delhi, INDIA.

Phone no: +91 8309660544 Email id: harikrishnaallu7@ gmail.com

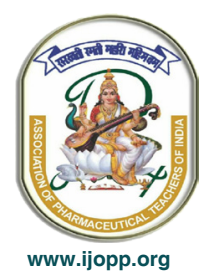


Information, Prescription Interpretation, Evolution, Implementation and Drug Administration. ${ }^{5}$ Their responsibility is very important in Out-Patient health care setting. Out-Patient Clinical Pharmacy Health care setting establishment is so important to provide Clinical Pharmacy Servises. ${ }^{3}$ The Clinical Pharmacy Services was utilizing globally as an Ambulatory care, Adverse Drug reaction monitoring, Patient Therapeutic goals; Clinical Pharmacist was the accountable person for Pharmacotherpeutic goals of Patient and Medication Safety in all Health Departments. ${ }^{6}$

\section{Establishment and Functional Ethics of Pharmacy Practice for Out-Patients in India}

Clinical establishment Act 2010 in India was described as a hospital, maternity home, nursing home, Dispensary, clinic, sanatorium or whatever named institute that was provides services as on. Dispensary is a part of Pharmacy Practice it was describing as analysis (Interpretation; Evaluation; Implementation) of medical orders; Dispensing of prescriptions, drug orders. Clinical Pharmacist need to participate in device and drug selection; drug administration; drug dosage regimen review of the patient; medication related research; providing patient counseling; need to participate where the Pharmaceutical care require in all areas of patient care including primary care.

Practicing Principles of Pharmacy Practice was:

i. Clinical Pharmacist was accountable Person for providing a Safety, Therapeutic Efficacy and Costeffective of Patient Prescription. It was work out by Interpretation, Evaluation, Implementation of the Prescription as per lab Investigation and patient Disease condition.

ii. Providing a Patient Medication and Disease Education is essential Responsibility of Clinical Pharmacist.

iii. Drug and Poison Information is a needful service for the Public, Health care Professionals, and these service responsibilities taken by the DIPh (Drug Information Pharacist).

iv. Provide cost-effective Prescription.

v. Health Promotion and Drug Administration.

vi. Medication relevant Research was professional responsibility of the Clinical Pharmacist. ${ }^{5}$
Needs of Clinical Pharmacist in society for Out-Patients

Pharma Doctor's Clinical Pharmacy Service is very need full aspect in Society for out Patient. Patient like Diabetes mellitus (DM), Hypertension (HTN), Coronary Heart Disease, Chronic Renal Failure (CRF), Thyroid Disease, Cancer, HIV, etc. like chronic Disease Patients have chance to associate with other multiple Diseases also, such patients need to use multiple treatments with multiple medication, those medication can have a chance to get interact with each other because of this Drug - Drug, Drug- lab, Drug- Disease, Drug- food, Drug- Alcohol interaction loses its therapeutic activity and Patient can have chance to confuse on multiple treatment medication administration, for overcome these barrier Patient need knowledge on medication administration, such like several therapeutic barriers need face by the multiple Diseases treated patients. ${ }^{8}$ Clinical Pharmacist was medication expert; he/she will provide the better service to overcome the Pharmacotherapeutic barriers by making the safest and therapeutic effective, Pharmacoeconamic cost-effective Prescription. Provide the medication adherence knowledge to the patient, its improve better medication administration and quality of life of patient. ${ }^{5}$

Now a day's allopathic medication usage is a part life for the public. In our country having number of Pharma companies, Number of Brands and Number of combination of Medication. Because of this Public have a chance to get confuse with medication due to lack of Knowledge on new combination medication administration for out-patient, overcome of these situation the Drug and Poison Information Centers are required in out Patient clinical Pharmacy Practice.

\section{Establishment of Clinical Pharmacy for out- Patients}

Pharmacy Practice means Interpretation; Evaluation and Implementation in medical orders and drug administration. ${ }^{5}$

Interpretation; Evaluation in medication order or Prescription means analysis of Drug relevant Problems (DRP) like Drug relevant interaction, Dosage and Frequency adjustment, Poly- Pharmacy, Cost-efficacy, Pharmacotherapy efficacy based on period of medication usage, such parameters identification in the prescription than evaluate severity of DRP and provide the proper advise to the authorized Prescription writer on DRP severity. Interpretation; Evaluation in prescription order improves the prescription safety and increase the life span of patient in better economic effect in both 
in-Patient, and Out-Patients. ${ }^{5,9}$ Implementation of medical order by identification of DRP and advising safest prescription to authorized practitioner is possible by the establishing of clinical pharmacy with support of proper documentation, usage of medication related medical software's with the knowledge of pharmacotherapy, Therapeutic drug monitoring and pharmacokinetic, Pharmacology, clinical pharmacy etc(as on Pharm.D syllabus). The important aspect is to provide a better Clinical Pharmacy Practice. Drug administration, patient counseling and Drug and Poison Information is also part of Clinical Pharmacy Practice. The establishment of clinical pharmacy for out-patient was need to require certain equipment's, Document registers and forms, medical software's and need to fame a separate setup at site of community pharmacy as per Pharmacy practice regulation 2015 (PPR 2015). ${ }^{5}$

\section{Services in clinical Pharmacy in Out-Patients}

- Prescription analysis as per PPR 2015.

- Patient Counseling on Medication, Disease and life style change.

- Drug and Poison Information.

- Medication related Research. ${ }^{5}$

- Conducting the Medication safety and Disease awareness programs.

\section{Equipment's}

Spigmomanometer, Stethoscope, Glucometer, Thermometer, Computer, Printer, land Phone, Projector, Internet connection.

Purpose: Spigmomanometer, Stethoscope, Glcometer, Thermometer was used for the Physical, Systemic, Blood glucose examination, such basic examination help us for proper patient prescription analysis. Computer, Printer, Projector, internet connection it will use for prescription analysis software, patient counseling leaflets, videos projection for proper understanding and drug information searching, land Phone will used for Drug information quarries from public, health care professionals.

\section{Record}

Prescription record (Appendix-II as per PPR2015), Patient visiting record, Reference Book maintenance record, Patient Disease Information Broachers and leaflets Record, Equipment record, Annual Clinical Pharmacy Activity Record.
Purpose: Records were used for maintenance of every Document and records data of Clinical activities. These data preservation will help us to improve pharma health care clinical activities.

\section{Forms}

Clinical Pharmacy activities was expressing through Documents like Prescription Record form (Appendix as per PPR 2015), Patient Counseling form, Drug and Poison Information requesting form, Drug and Poison Documentation form, Clinical Pharmacist Advising report.

Purpose: These Documents was exhibits clinical Pharmacist need in Health care system. These using for preserving the patient data for reference for Clinical Pharmacy Health care activities.

\section{Medical Software}

Prescription analysis and medication data software, Patient data storage software.

Purpose: Medication data software was one of the data tool for improving the safest, effective prescription and analysis software help to find the chemical related reaction with other administration medication, food, Medication induced lab investigational error, Disease related obstruction of medication efficacy. Patient data software help us to preserve the patient data for long time to help use know about patient condition for further Disease complication treatment.

\section{Reference Books}

Medication data reference books (Martindale the Complete Drug Reference, ASHP, Indian National formulary, British National formulary, Indian Pharmacopeia (IP), British Pharmacopeia (BP), United state Pharmacopeia (USP)), Drug Interaction books (Stockley Drug Interaction), Disease related Guidelines, journals.

Purpose: These books will help us to make evidence based prescription as safest and effective to the patient.

\section{Miscellaneous things}

Need to maintain different tools to improve patient medication adherence, storage condition and understanding of illiterate out-patient medication administration. Need to maintain some instruments 
for identification of instant physiological and systemic investigational parameters for making a safest and effective Prescription.

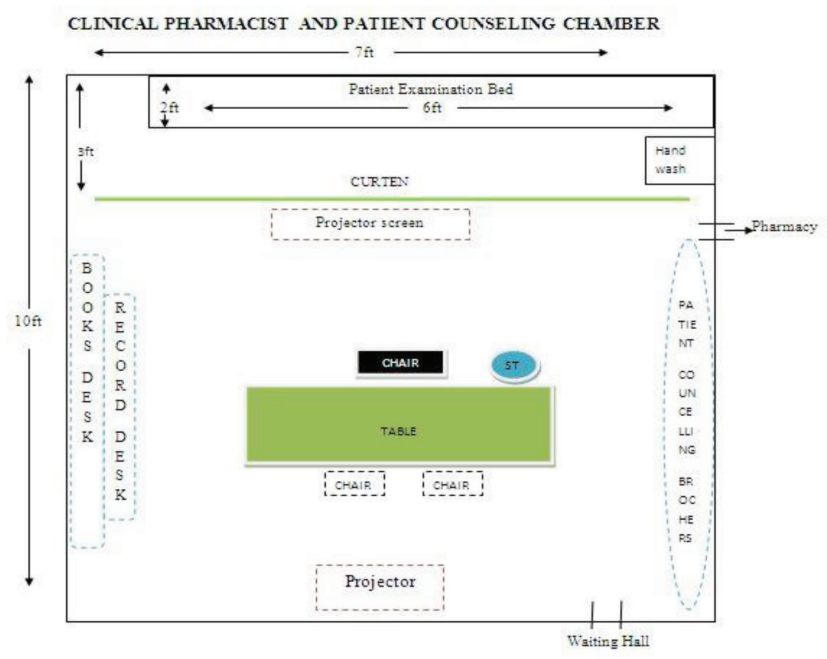

\section{Advantages}

1. Patient will get safest, therapeutic-effective and cost- effective treatment.

2. Patent will get good Knowledge on medication usage and it adherence, Disease Knowledge its help to change the life style for cure the Disease.

3. Minimize the medication usage burden on the patient.

4. Patients, public and Health care Professional will get medication related Evidence based Information.

5. Poor people will get basic Diagnostic investigation (Sugar test, Cholesterol test, Body mass Index (BMI) test, etc.).

6. Out - Patients need to get medication administration service and Knowledge.

7. Patient will get Knowledge of Diet based on Disease Condition and medication Administration.

Patient need to aware on medication side and adverse reaction.

\section{CONCLUSION}

Clinical Pharmacy is the part of Pharmacy Practice. Clinical Pharmacy practicener was called as a Clinical Pharmacist. Clinical Pharmacy services was needed in out-patient also as well as in-patient Clinical Pharmacy which is establish in the Hospital Pharmacy same as it was need to establish in site of Community Pharmacy for providing the safest, effective, better economic-effective medication to the out-patient for full pledged therapy. Out patients like DM, HTN, CHD, CRD, Thyroid Disease patients was spending more in-pocket economy for on their therapy but some of the patient cannot getting proper cure due to DRPs, Avoiding of these situation need Practice Clinical Pharmacy services in the site of Community Pharmacy for out-Patients. Patient need to get knowledge about medication administration, medication adherence, medication storage, Disease and its life style changes for proper cure. Drug and Poison information centre very necessary parameter in Clinical Pharmacy Service for giving the Drug related Information in form of Patient based and Knowledge base to the Health care professionals, Public, Patients for better usage of Medication. Clinical Pharmacy services is need to implement in Community Pharmacy setting based on Pharmacy practice regulation 2015 (PPR). Dispense means not only supplying of Medication it should Implementation and evolution of Prescription order it means to observing and avoiding the DRPs from the prescription, if any changes need in prescription about Medication active Ingredient that should be advise to the prescriber who is having prescription order writing authority as per Drug and Cosmetic Act. 1940. In a Health care Sector Better Disease cure will get possible when the both Diagnosis and Treatment was effective and safest. It will provide cost- effective health care Service.

"Better Disease cure $=$ effective Diagnosis + Safest and effective Treatment.

Cost- effective health care service $=$ effective Diagnosis

+ Safest and effective Treatment.”

\section{ACKNOWLEDGEMENT}

Review Article was prepared by the support of Doctor of Pharmacy Association team and other Pharm.D graduates and association support. It was completely dedicated to Doctor of Pharmacy graduates, who is Practicing Clinical Pharmacy for Out-Patients.

\section{CONFLICT OF INTEREST}

This article was prepared for the implementation of Pharmacy Practice regulation 2015 in the Out-Patient health care system. This service will avoid the DRP in Out-Patient Prescription as well as provide the safest, therapeutic effective and cost-effective medication to the Out-Patient. 


\section{ABBREVIATIONS}

BMI: Body mass Index; CHD: Chronic Heart Disease; CRF: Chronic Renal Failure; Pharm.D: Doctor of Pharmacy; DIPh: Drug Information Pharmacist; DM: Diabetes mellitus; DRP: Drug relevant Problems; HIV: Human Immune deficiency Virus; HTN: Hypertension; PCI: Pharmacy Council of India; PPR 2015: Pharmacy Practice Regulation 2015.

\section{REFERENCES}

1. Doctor of Pharmacy Regulation 2008 under section 10 Pharmacy Act 1948.
2. Maqbool Mudasir, Dar Mohmad Amin, Rasool Shafiqa, Mir Suhail Ahmad, Bhat Ahsan Ullaha. Clinical Pharmacy Practice in health care system, ejpmr. 2019;6(4):630-3.

3. Thomas Dixon, book Clinical Pharmacy Education. Practice and research: positioning clinical pharmacy in Pharmacy Practice, Elsevier, January 2020, $1-3$.

4. India pharmacy practice regulations, Chapter-I. Section 2; 2015, Pag-28.

5. India practice regulations; 2015.

6. Jacobi Judith, ACCP. MCCM, BCPS, BCCCP, Clinical Pharmacists: practitioners who are essential members of your clinical care team. rev Med Clin Condes. 2016;27(5):571-7.

7. Clinical establishment act 2010

8. Oliva Elizabeth M., Maisel Natalya C., Gordon Adam J., Harris Alex H.S. Barriers to use of pharmacotherapy for addiction disorders and how to overcome them. Curr Psychiatry Rep. 2011;13(5):374-81. doi: 10.1007/s11920-011-0222-2, PMID 21773951.

9. Solanki Nilay D, Shah Chaital. Prescription audit in outpatient department of multispecialty hospital in western India: an observational study. Int J Clin Trials. 2015 February;2(1):14-9. doi: 10.5455/2349-3259.ijct20150203. 